\title{
Method of determining potential barrier heights at submonolayer AlAs/GaAs heterointerfaces
}

\author{
Gil-Ho Kim \\ Telecommunication Basic Research Laboratory, ETRI, Yusong P.O. Box 106, Taejon 305-600, Korea \\ and Cavendish Laboratory, Madingley Road, Cambridge CB3 OHE, United Kingdom \\ M. Y. Simmons \\ Semiconductor Nanofabrication Facility, School of Physics, University of New South Wales, Sydney 2052, Australia \\ C.-T. Liang \\ Department of Physics, National Taiwan University, Taipei 106, Taiwan
}

D. A. Ritchie and A. C. Churchill*

Cavendish Laboratory, Madingley Road, Cambridge CB3 OHE, United Kingdom

H.-S. Sim and K. J. Chang

Department of Physics, Korea Advanced Institute of Science and Technology, Taejon 305-701, Korea

G. Ihm and N. Kim

Department of Physics, Chungnam National University, Taejon 305-764, Korea

(Received 20 July 2001; published 5 October 2001)

\begin{abstract}
We report low-field magnetoresistance measurements of a two-dimensional electron gas formed in a GaAs quantum well, in which half a monolayer of AlAs has been inserted into the center of the well. A large anisotropy is observed in both the mobility and the low field magnetoresistance in the orthogonal [ $\overline{1} 10]$ and [110] directions. We describe a method of using the anisotropic low field magnetoresistance to calculate the magnitude of the effective potential of the AlAs submonolayer at the GaAs/AlGaAs heterointerface.
\end{abstract}

DOI: $10.1103 /$ PhysRevB.64.165313

PACS number(s): 73.21.-b, 73.40.-c, 73.61.-r, 73.50.Jt

The ability to control the electronic potential at a semiconductor heterostructure interface is important for studies of systems such as lateral superlattices, one-dimensional (1D) quantum wires and single quantum wells. A standard method of controlling the potential at a heterostructure interface is to use a metallic Schottky gate on the surface of the device produced by a technique such as electron beam lithography (EBL). A good example of this is the transport properties of a $1 D$ periodic potential formed by the use of a grating gate fabricated using EBL, which has attracted much interest both experimentally $^{1-4}$ and theoretically. ${ }^{5-7}$ Another potentially significant route for the fabrication of one-, 8,9 and zerodimensional $^{10,11}$ nanostructures is by natural formation during the growth procedure. Whilst there are a few studies of the physics of such systems ${ }^{12-16}$ there is a notable dearth in the literature of detailed magnetotransport measurements of naturally formed submonolayer potential heterostructures.

In this paper we present detailed magnetotransport studies of a two-dimensional electron gas (2DEG) in a GaAs quantum well in which half a monolayer of AlAs has been inserted into the center of the well. Using a full surface Schottky gate it is possible to control the carrier density of the 2DEG in the well and observe the effect of the AlAs potential on the magnetoresistance in both the [110] and [110] directions. Previous studies of the magnetoresistance of a 2 DEG with a periodic potential, created with a Schottky grating gate, showed that electrons are trapped in potential energy minima below a critical magnetic field. ${ }^{2-4}$ By altering the voltage on the grating gate it was possible to control the potential energy at the heterointerface and thereby tailor the electrical properties of the 2DEG. In our devices we measure the magnetoresistance characteristics of a naturally formed 1D periodic potential with a fixed effective potential energy due to the incorporation of a submonolayer of AlAs. The results demonstrate the anisotropic nature of this fixed 1D periodic potential associated with the formation of elongated AlAs islands in the [ $\overline{1} 10]$ direction within the GaAs quantum well.

Figure 1(a) shows a schematic cross-sectional illustration of our device, a $n$-AlGaAs/GaAs heterojunction grown by molecular beam epitaxy on an undoped GaAs (001) substrate deliberately misoriented by $0.09^{\circ}$. The structure consists of a $0.6 \mu \mathrm{m}$ thick undoped GaAs buffer layer, followed by a $500 \AA$ undoped $\mathrm{Al}_{0.33} \mathrm{Ga}_{0.67} \mathrm{As}$ barrier, a $200 \AA$ undoped GaAs quantum well, a $400 \AA$ undoped $\mathrm{Al}_{0.33} \mathrm{Ga}_{0.67} \mathrm{As}$ spacer layer, a $400 \AA$ Si-doped $\left(1 \times 10^{18} \mathrm{~cm}^{-3}\right) \quad \mathrm{Al}_{0.33} \mathrm{Ga}_{0.67} \mathrm{As}$ layer, and finally a $170 \AA$ GaAs capping layer. Results are presented from two different samples. In sample A, an AlAs layer with a coverage of approximately 0.5 monolayers (ML) was inserted into the GaAs quantum well using the migration enhanced step flow growth mode described in Ref. 8. Sample $\mathrm{B}$ is a reference sample with the same heterostructure design grown on an identically misoriented (001) GaAs wafer but without the AlAs submonolayer insertion.

The devices were processed into an orthogonal Hall bar geometry in Fig. 1(c), with the current in either the [110] and [ 110$]$ direction, with a width of $80 \mu \mathrm{m}$ and a length of 
(a)

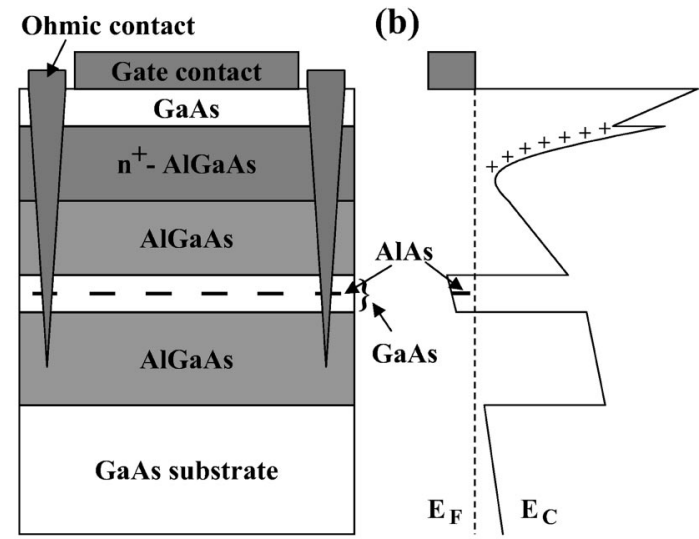

(c)

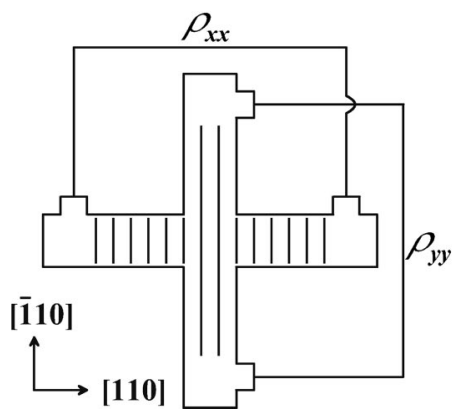

FIG. 1. (a) Structure of the samples. (b) Schematic of the conduction-band profile in the growth $(z)$ direction, at the site of AlAs deposition. (c) Schematic illustration showing the orthogonal Hall bar geometry. Measurements were made by four terminal configurations defined magnetoresistance $\rho_{x x}$ and $\rho_{y y}$ when the constant current flows in the [110] and [ $\overline{1} 10]$ direction, respectively.

$800 \mu \mathrm{m}$. Ohmic contacts were made by thermal evaporation of AuGeNi alloys. A transparent gate was constructed onto the top of the structure by $\mathrm{NiCr} / \mathrm{Au}$ to control the carrier density $n_{s}$ in the 2DEG channel. All measurements were taken after illumination with a red-light-emitting diode at a temperature of $1.5 \mathrm{~K}$ and a constant source-drain current of $100 \mathrm{nA}$ at $73 \mathrm{~Hz}$. The magnetoresistance was measured using standard four-terminal ac phase sensitive techniques.

Using a GaAs substrate with an intentional misorientation from the (001) plane, it is possible to form a periodic potential of AlAs islands along the [110] surface using migration enhanced epitaxy. ${ }^{17}$ With transmission electron microscopy images ${ }^{18}$ on a similar substrate, from the same batch of wafers, it was possible to determine the angle of misorientation, $\alpha$ from the (001) plane as $\alpha \approx 0.09^{\circ} .{ }^{19}$ Knowing this angle and the thickness of one monolayer of GaAs, it is possible to calculate the period of the effective corrugated potential from $d=a \cot \alpha \approx 180 \mathrm{~nm}$.

Figures 2(a) and 2(b) show the low-field magnetoresistance of the 2DEG in device A for the [110] and [1 10] directions, respectively. The measurements were taken at 1.5 $\mathrm{K}$ at a carrier density of $2.34 \times 10^{11} \mathrm{~cm}^{-2}\left(V_{g}=0\right)$ estimated from the slope of the Hall resistance for both directions. The zero-field resistance in the [110] direction is about one-and-a-half times higher than that in the [110] direction leading to a marked anisotropy in the mobility in the two
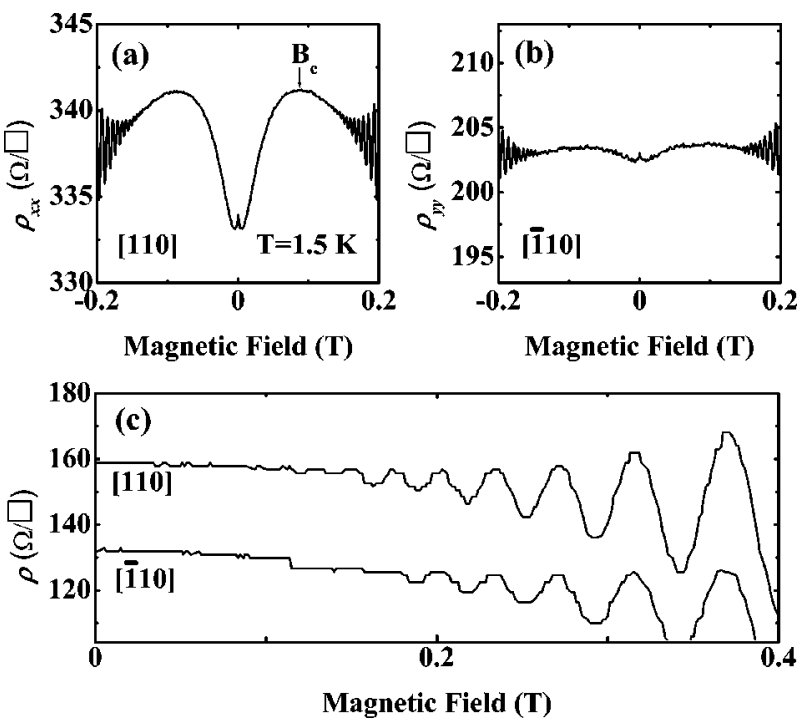

FIG. 2. Low-field magnetoresistivity traces for sample A at a carrier density of $2.3 \times 10^{11} \mathrm{~cm}^{-2}$ in the (a) [110] and (b) [110] directions and (c) low field magnetoresistivity traces for sample $\mathrm{B}$ at a carrier density of $2.6 \times 10^{11} \mathrm{~cm}^{-2}$ in both orthogonal directions. All traces are at $1.5 \mathrm{~K}$.

orthogonal directions (with a peak mobility of 1.2 $\left.\times 10^{5} \mathrm{~cm}^{2} / \mathrm{V} \mathrm{s}\right)$. At very low magnetic fields $(<0.02 \mathrm{~T})$ a small peak around zero field is observed in both directions due to the suppression of the weak localization effect. ${ }^{20}$ At higher fields a positive magnetoresistance is observed in both directions but which is much more pronounced in the [110] direction. The occurrence of a strong magnetoresistance in the [110] direction is discussed in more detail below. At even higher fields $(B>0.15$ T) Shubnikov-de Haas oscillations are observed.

Figure 2(c) shows the low-field magnetoresistance for both the $[110]$ and $[\overline{1} 10]$ directions for the reference sample B. The measurements were taken at a similar carrier density of $2.58 \times 10^{11} \mathrm{~cm}^{-2}$ in both directions and immediately we can see that the absence of scattering from the inserted AlAs islands leads to a much higher maximum mobility (1.8 $\times 10^{5} \mathrm{~cm}^{2} / \mathrm{V} \mathrm{s}$ ) for this sample. At this density the ratio of the anisotropic resistance is $20 \%$ in the two orthogonal directions for the reference sample B in contrast to $63 \%$ for sample A. It is well known that for 2DEG's grown on the (001) surface of GaAs the mobility in the [110] direction can be higher than that in the [110] direction, under certain growth conditions, due to the formation of elongated islands at the GaAs/AlGaAs interface. ${ }^{21}$ However, any potential barrier due to the island formation at this heterointerface is sufficiently small that it does not manifest itself as a difference in the low field magnetoresistance in the two orthogonal directions. We note that no positive magnetoresistance is observed for either direction in the reference sample B.

The main result of this paper concerns the difference in the low field magnetoresistance in the two orthogonal directions due to the formation of elongated AlAs islands in the GaAs quantum well. The absence of a positive magnetoresistance in the reference sample B clearly demonstrates that 

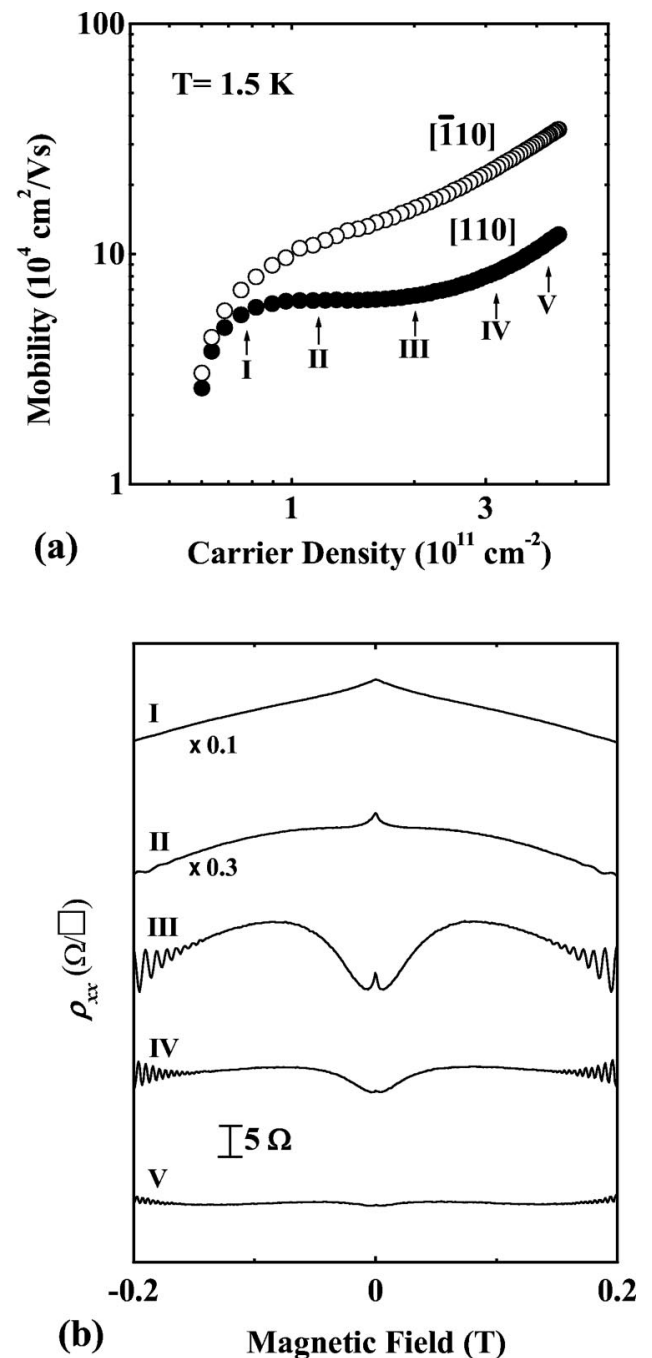

FIG. 3. (a) The mobility along the [110] and [110] directions for sample A as a function of carrier density $n_{s}$ at $T=1.5 \mathrm{~K}$. Arrows with Roman numerals indicate the points at which the magnetoresistance data in Fig. 3(b) were taken. (b) Magnetoresistance in the [110] direction are measured at gate voltages -0.20 (top), $-0.15,-0.05,0.10$, and $0.25 \mathrm{~V}$ (bottom), respectively.

the observed anisotropic positive magnetoresistance in the AlAs inserted sample $\mathrm{A}$ is due to the formation of an induced quasi-1D lateral superlattice.

Figure 3(a) shows the mobility $\mu$ versus the carrier density $n_{s}$ at $1.5 \mathrm{~K}$ in the [110] and [ $\left.\overline{1} 10\right]$ directions for sample A. At low densities $\left(0.7 \times 10^{11} \mathrm{~cm}^{-2}\right)$ the mobility is independent of direction as the electrons are mainly scattered by isotropic impurity scattering. At higher densities anisotropic interface scattering due to the quasiperiodic 1D potential dominates and the mobility becomes anisotropic. In order to provide further insight as to what is happening at the different carrier densities (marked I-V) we present the corresponding magnetoresistance traces along the [110] direction in Fig. 3(b).

At low carrier densities $\left(<0.68 \times 10^{11} \mathrm{~cm}^{-2}\right)$ where the mobility is isotropic, a large negative magnetoresistance is observed [see Fig. 3(b) at $V_{g}=-0.20$ V]. Similar results

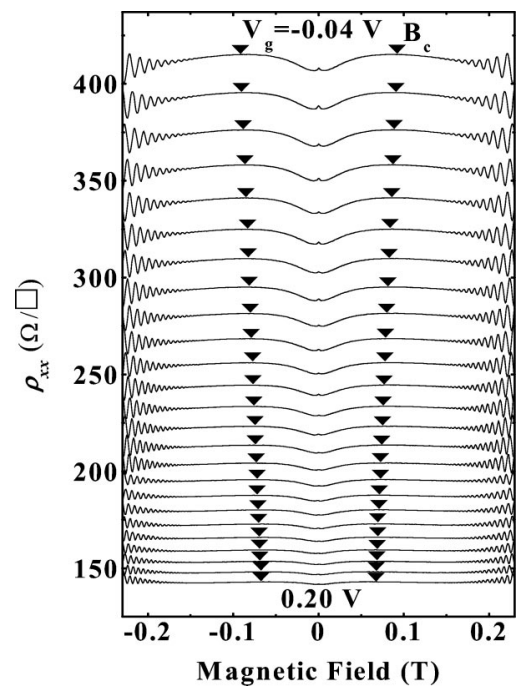

FIG. 4. The magnetoresistivity $\rho_{x x}$ in the [110] direction for sample A for a set of gate voltages from -0.04 to $0.20 \mathrm{~V}$ (stepped in units of $0.01 \mathrm{~V}$ ) for $T=1.5 \mathrm{~K}$. The triangles indicate $B_{c}$ of magnetic breakdown.

have been obtained in heterostructures where a monolayer of self-assembled InAs quantum dots has been inserted in a 2DEG of similar mobility. ${ }^{22}$

As the carrier density is increased interface roughness scattering starts to dominate and a large positive magnetoresistance develops, with a maxima at $B=B_{c}$ (see III). In order to understand the occurrence of a large positive magnetoresistance observed in the [110] direction for the intermediate field range ( 0.02 to $0.2 \mathrm{~T})$ we turn to an earlier study of $1 \mathrm{D}$ surface superlattices by Beton et al. ${ }^{2,3}$ They observed a positive magnetoresistance in a periodically modulated 2DEG when the direction of the current flow is orthogonal to the superlattice axis. The magnetoresistance shows a maxima at a critical field $B_{c}$, where $B_{c}$ is given by

$$
B_{c}=2 \pi V_{\mathrm{eff}} / d v_{F},
$$

where $V_{\text {eff }}$ is the amplitude of the effective potential, $d$ is the period, and $v_{F}$ is the Fermi velocity. The positive magnetoresistance arises from electron orbits "streaming" along the 1D superlattice potential. When the force due to the magnetic field is greater than that due to the electric field $\left(B>B_{c}\right)$ a form of magnetic breakdown occurs and the streaming orbits are suppressed. This is a purely classical effect which ignores the effect of tunneling through the AlAs potential barriers. In our case the periodic potential arises from the AlAs superlattice, and from Fig. 2(a) we can see that $B_{c} \approx 0.086 \mathrm{~T}$. Using Eq. (1) we can estimate the effective periodic potential of the AlAs, which is $V_{\text {eff }} \approx 0.516 \mathrm{meV}$. Increasing the carrier density even further (traces IV and V) causes the magnitude of the positive magnetoresistance to decrease. This is because the Fermi energy increases with increasing carrier density, so the relative height of the fixed potential due to the periodic 1D AlAs superlattice decreases.

Figure 4 shows in more detail the gradual decrease in the magnitude of the positive magnetoresistance along the [110] direction from a gate voltage of -0.04 to $0.20 \mathrm{~V}$ (over which 
the carrier density is varied from $2.02 \times 10^{11}$ to 3.84 $\times 10^{11} \mathrm{~cm}^{-2}$ at $T=1.5 \mathrm{~K}$ ). It is possible to derive the change in the magnitude of the positive magnetoresistance semiclassically as follows. Taking into account the anisotropy in the mobilities in the two orthogonal directions, the unperturbed diffusion tensor for $2 \mathrm{D}$ electron systems in a magnetic field is easily obtained as $D_{x x}^{0}=\tau_{x} g, D_{y y}^{0}=\tau_{y} g$, and $D_{y x}^{0}=D_{x y}^{0}=\omega_{c} \tau_{x} \tau_{y} g$, where $\tau_{x}$ and $\tau_{y}$ are the scattering times in [110] and [110] directions, respectively, and $g$ $=\frac{1}{2} v_{F}^{2} /\left(1+\omega_{c}^{2} \tau_{x} \tau_{y}\right)$. In a magnetic field, the effective corrugated potential $V_{\text {eff }}$ induces electrons to stream along the [110] direction, ${ }^{2}$ however, the quasiperiodic potential $V_{\text {eff }}$ means that the electrons also drift in the [110] direction. The observation of a positive magnetoresistance with a corresponding magnetic breakdown in $\rho_{y y}$, shown in Fig. 2(b), support this assumption. The electron drift in each of the two directions results in an additional electron diffusion given by $\delta D_{x x}=\tau_{x}\left\langle v_{d, x}^{2}\right\rangle$ and $\delta D_{y y}=\tau_{y}\left\langle v_{d, y}^{2}\right\rangle$, respectively, where $v_{d}$ is a drift velocity. ${ }^{5}$

From the Einstein relation between the resistivity tensor and the diffusion tensor, the longitudinal resistivity $\rho_{x x}$ satisfies $\rho_{x x}=\left(h^{2} / 4 \pi m e^{2}\right) D_{y y} /\left(D_{x x} D_{y y}-D_{x y}^{0} D_{y x}^{0}\right)$, where $D_{x x}=D_{x x}^{0}+\delta D_{x x}$ and $D_{y y}=D_{y y}^{0}+\delta D_{y y}$. Since $V_{\text {eff }}$ does not provide an ideal potential we assume $\left\langle v_{d, x}^{2}\right\rangle,\left\langle v_{d, y}^{2}\right\rangle \ll v_{F}^{2}$. Note that $\omega_{c} \tau_{y}$ is of the order of 1 at $B_{c}$, hence the assumption $\omega_{c} \tau \gg 1$ in Ref. 2 is not strictly applicable at $B>B_{c}$ in our work. To leading order in $\left\langle v_{d, x}^{2}\right\rangle / v_{F}^{2}$ and $\left\langle v_{d, y}^{2}\right\rangle / v_{F}^{2}$, we obtain

$$
\begin{aligned}
\Delta \rho_{x x} & =\rho_{x x}-\rho_{x x}^{(0)} \\
& =2 \rho_{x x}^{(0)}\left(\omega_{c}^{2} \tau_{x} \tau_{y} \frac{\left\langle v_{d, y}^{2}\right\rangle}{v_{F}^{2}}-\frac{\left\langle v_{d, x}^{2}\right\rangle}{v_{F}^{2}}\right) .
\end{aligned}
$$

Here, $\rho_{x x}^{(0)}\left[=h^{2} /\left(2 \pi m_{e}^{*} e^{2} \tau_{x} v_{F}^{2}\right)\right]$ is the unperturbed longitudinal resistivity. The first term in Eq. (3) is attributed to the streaming orbits and $\left\langle v_{d, y}^{2}\right\rangle$ depends on the ratio of the number of streaming electron orbits in the [110] direction divided by the total number of orbits. The electron drift in the [110] direction gives rise to the second term in Eq. (3) which reduces the resistivity in the [110] direction. The dependence of $\left\langle v_{d, x}^{2}\right\rangle$ on the magnetic field is similar to that of $\left\langle v_{d, y}^{2}\right\rangle ;\left\langle v_{d, x}^{2}\right\rangle$ decreases as the magnetic field increases. ${ }^{2}$ Since the effective potential of the AlAs submonolayer is more periodic in the [110] direction than in the [110] direction, $\left\langle v_{d, y}^{2}\right\rangle>\left\langle v_{d, x}^{2}\right\rangle$. Thus, for samples of higher mobility, the first term in Eq. (3) is more important than the second; giving rise to the positive magnetoresistance at low magnetic fields and magnetic breakdown at a critical field, as shown in Fig. 4.

As seen from Eq. (1), knowing $B_{c}$ and $n_{s}$ we can determine the effective $1 \mathrm{D}$ periodic potential energy of the AlAs submonolayer (shown in Table I) at each different carrier density. It can be seen that the effective potential $V_{\text {eff }}$ of AlAs is almost constant over all this range of densities. This data is also plotted in Fig. 5, where the critical field $B_{c}$ taken from
TABLE I. Gate voltages $\left(V_{g}\right)$ and critical magnetic-field $\left(B_{c}\right)$ values for the peak in the magnetoresistance for sample A. The Fermi energy $E_{F}$ is derived from the carrier density $n_{s}$, while $V_{\text {eff }}$ is calculated from $B_{c}$ and $n_{s}$, using Eq. (1).

\begin{tabular}{lcccc}
\hline \hline$V_{g}(\mathrm{~V})$ & $n_{s}\left(\times 10^{11} \mathrm{~cm}^{-2}\right)$ & $E_{F}(\mathrm{meV})$ & $B_{c}(\mathrm{~T})$ & $V_{\text {eff }}(\mathrm{meV})$ \\
\hline-0.04 & 2.02 & 7.2 & 0.092 & 0.513 \\
0 & 2.34 & 8.3 & 0.086 & 0.516 \\
0.04 & 2.66 & 9.5 & 0.080 & 0.512 \\
0.12 & 3.28 & 11.7 & 0.072 & 0.512 \\
0.20 & 3.84 & 13.7 & 0.067 & 0.515 \\
0.28 & 4.28 & 15.3 & 0.065 & 0.528 \\
\hline \hline
\end{tabular}

experimental points in Fig. 4, is plotted as a function of the inverse period $1 /\left(d v_{F}\right)$. From the linear fit we can again see that the effective potential of the AlAs submonolayer is $\approx 0.512 \mathrm{meV}$. At low electron densities, $V_{\text {eff }}$ is observed to increase and deviate from $0.512 \mathrm{meV}$. This increase in $V_{\text {eff }}$ at low densities can be understood since the electron drift in the [100] direction, i.e., the second term in Eq. (3), becomes more important in lower-mobility samples, as compared with higher-mobility samples. If this second term is not neglected, as might be the case for lower-mobility samples, the critical fields may be shifted to higher values since $\left\langle v_{d, x}^{2}\right\rangle$ is a decreasing function of $B$ near $B_{c}$. Therefore, the estimated values of $V_{\text {eff }}$ obtained by fitting the slope should always be interpreted as a lower limit.

In summary, we have incorporated half a monolayer of AlAs into a GaAs quantum well to induce an imperfectly periodic $1 \mathrm{D}$ potential in a $2 \mathrm{DEG}$. The $2 \mathrm{DEG}$ mobility is observed to be anisotropic and carrier density dependent. At densities above $0.68 \times 10^{11} \mathrm{~cm}^{-2}$ the mobility in the [110] direction is observed to be higher than in the [110] direction. A strong low-field positive magnetoresistance is observed in the [110] direction, with a corresponding critical magnetic breakdown field. From the theory of magnetic breakdown we can calculate the effective potential of the AlAs submonolayer as a function of carrier density and found that the potential is fixed at $0.512 \mathrm{meV}$. The results demonstrate a method of inducing a fixed periodic potential at a heterostructure interface and using electrical transport measurements to determine the effective height of potential.

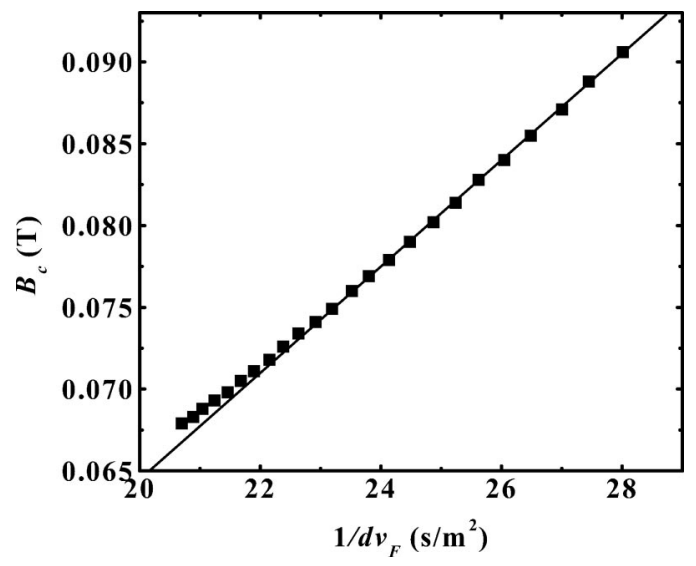

FIG. 5. $B_{c}$ as a function of $1 / d v_{F}$. 
The authors would like to thank P.H. Beton and P.C. Main for helpful discussions. We wish to thank the Engineering and Physical Sciences Research Council (UK) for supporting this work. G.H.K. acknowledges financial support from the
Korean Ministry of Information and Communication and D.A.R. acknowledges support from Toshiba Research Europe, Ltd. C.T.L. is grateful for support from NSC, Taiwan, and the Research Council, National Taiwan University.
*Also at: Defense Science and Technology Laboratory, St Andrews Road, Great Malvern, Worcestershire WR14 3PS, UK.

${ }^{1}$ D. Weiss, K.v. Klitzing, K. Ploog, and G. Weimann, Europhys. Lett. 8, 179 (1989).

${ }^{2}$ P.H. Beton, E.S. Alves, P.C. Main, L. Eaves, M.W. Dellow, M. Henini, O.H. Hughes, S.P. Beaumont, and C.D.W. Wilkinson, Phys. Rev. B 42, 9229 (1990).

${ }^{3}$ P.H. Beton, M.W. Dellow, P.C. Main, E.S. Alves, L. Eaves, S.P. Beaumont, and C.D.W. Wilkinson, Phys. Rev. B 43, 9980 (1991).

${ }^{4}$ J. Takahara, S. Yoshimatsu, K. Gamo, S. Namba, S. Takaoka, and K. Murase, Jpn. J. Appl. Phys. 33, 3837 (1994).

${ }^{5}$ C.W.J. Beenakker, Phys. Rev. Lett. 62, 2020 (1989).

${ }^{6}$ J. Kučera, P. Středa, and R.R. Gerhardts, Phys. Rev. B 55, 14439 (1997).

${ }^{7}$ R. Menne and R.R. Gerhardts, Phys. Rev. B 57, 1707 (1998).

${ }^{8}$ J. Motohisa, M. Tanaka, and H. Sakaki, Appl. Phys. Lett. 55, 1214 (1989).

${ }^{9}$ Y. Nakamura, S. Koshiba, and H. Sakaki, Appl. Phys. Lett. 69, 4093 (1996).

${ }^{10}$ B.D. Min, Y. Kim, E.K. Kim, S. Min, and M.J. Park, Phys. Rev. B 57, 11879 (1998).
${ }^{11}$ G.D. Lian, J. Yuan, L.M. Brown, G.H. Kim, and D.A. Ritchie, Appl. Phys. Lett. 73, 49 (1998).

${ }^{12}$ E.A. Kondrashkina, S.A. Stepanov, R. Opitz, M. Schmidbauer, R. Köhler, R. Hey, M. Wassermeier, and D.V. Novikov, Phys. Rev. B 56, 10469 (1997).

${ }^{13}$ M.D. Ventra and A. Baldereschi, Phys. Rev. B 57, 3733 (1998).

${ }^{14}$ T. Mélin and F. Laruelle, Phys. Rev. Lett. 81, 4460 (1998).

${ }^{15}$ K. Ensslin, S.A. Chalmers, P.M. Petroff, A.C. Gossard, and H. Kroemer, Superlattices Microstruct. 9, 119 (1991).

${ }^{16}$ T. Noda, J. Motohisa, and H. Sakaki, Surf. Sci. 267, 187 (1992).

${ }^{17}$ M. Tanaka and H. Sakaki, Appl. Phys. Lett. 54, 1326 (1989).

${ }^{18}$ J. Yuan, G. D. Lian, G. H. Kim, D. A. Ritchie, Z. Y. Li, and L. M. Brown (unpublished).

${ }^{19}$ Gil-Ho Kim, D.A. Ritchie, C.-T. Liang, G.D. Lian, J. Yuan, M. Pepper, and L.M. Brown, Appl. Phys. Lett. 78, 3896 (2001).

${ }^{20}$ R.A. Davies, M.J. Uren, and M. Pepper, J. Phys. C 14, L531 (1981).

${ }^{21}$ T. Saku, Y. Horikoshi, and Y. Tokura, Jpn. J. Appl. Phys. 35, 34 (1996).

${ }^{22}$ G.H. Kim, D.A. Ritchie, M. Pepper, G.D. Lian, J. Yuan, and L.M. Brown, Appl. Phys. Lett. 73, 2468 (1998). 